\title{
MOTIVES FOR AND BARRIERS TO EXERCISING ACROSS TRIMESTERS OF PREGNANCY IN HEALTH CLUB MEMBERS
}

original paper

( ) University School of Physical Education in Wroclaw

DOI: https://doi.org/10.5114/hm.2020.91342

\author{
ERIN WHITE ${ }^{1}$, KARIN PFEIFER ${ }^{2}$, CLAUDIA HOLZMAN ${ }^{2}$, JAMES PIVARNIK $^{2}$ \\ ${ }^{1}$ Winona State University, Winona, USA \\ ${ }^{2}$ Michigan State University, East Lansing, USA
}

\begin{abstract}
Purpose. Determining the motives for and barriers to physical activity (PA) during pregnancy may help to promote PA throughout gestation. Therefore, the purpose of this study was to describe the most frequent motives for and barriers to maternal exercise by trimester in exercising and non-exercising women who belonged to a health club.

Methods. A retrospective, online survey was available to the participating women. They stated the top 3 motives and barriers for exercise during each trimester. Frequencies and chi-squared test proportions were calculated.

Results. The study involved 295 women. The most commonly reported motives for exercise during each trimester was to stay healthy $(37.4 \%, 35.5 \%$, and $32.9 \%$, respectively) and to control weight $(19.8 \%, 27.1 \%$, and $25.5 \%$, respectively). The most commonly reported barrier to exercise was lack of energy/tiredness in all trimesters $(27.0 \%, 18.9 \%$, and $19.4 \%$, respectively). During trimesters 1 and 3, trimester-specific barriers such as pregnancy-related illness (18.5\%) and exercise being too uncomfortable (17.2\%) emerged, respectively.

Conclusions. PA motives and barriers did not change statistically significantly by trimester regardless of exercise status $(p>0.05)$. Although not explored in this study, tailoring PA messages to overcome the most salient barriers in each trimester, such as education regarding how to modify PA in the third trimester to make exercise more comfortable, may be more effective than promoting general health benefits of PA during pregnancy.
\end{abstract}

Key words: gestation, physical activity, motivation, women

\section{Introduction}

Pregnancy is a unique and opportune time in a woman's life to discuss healthy lifestyle behaviours such as physical activity (PA). Women are often more receptive to behavioural changes during pregnancy if they believe those changes will positively impact on the growing foetus. It is well-known that the benefits of PA during pregnancy far outweigh potential concerns [1]. Maternal benefits of regular PA during pregnancy include weight control [2] and reduction of backaches, constipation, and sleeping difficulties [3, 4]. In addition, women who are physically fit are able to better handle the physical demands placed upon them during pregnancy [5-7]. While the benefits of PA during pregnancy are well-documented, many women do not meet the recommendations and are less active than their non-pregnant counterparts [8]. It has been documented that $60 \%$ of women engaged in no leisure-time PA during pregnancy [9] despite the many health benefits for the mother and foetus. Even among active women, PA generally declines throughout gestation, especially in the third trimester [8-10]. This decline is, in part, due to various physical and physiological changes that occur from first to third trimesters, including, but not limited to, weight gain leading to changes in centre of gravity [11] and changes in hormone levels that can cause nausea and other feelings of illness [12]. Thus, as Fell et al. [13] conclude, pregnancy, in itself, is an event that leads to decreased PA levels across a variety of activity domains, but the largest changes are seen as women decrease participation in sport and exercise during pregnancy. Due to these changes, many women choose to decrease or

Correspondence address: Erin White, Winona State University, 175 W Mark Street Winona, Minnesota, 55987 USA, e-mail: ewhite@winona.edu

Received: June 4, 2019

Accepted for publication: October 28, 2019

Citation: White E, Pfeifer K, Holzman C, Pivarnik J. Motives for and barriers to exercising across trimesters of pregnancy in health club members. Hum Mov. 2020;21(3):21-30; doi: https://doi.org/10.5114/hm.2020.91342. 
E. White, K. Pfeifer, C. Holzman, J. Pivarnik, Exercise motives and barriers in pregnancy

stop being active rather than modifying their exercise routines to accommodate their changing body. Therefore, studying the reasons why women either choose to remain active or not during pregnancy is important. Furthermore, studying motives and barriers by trimester is essential as the mother and foetus undergo many changes physically which can alter a mother's ability and perception of ability to exercise.

Currently, it is not well known what motivates women to initiate or maintain PA throughout gestation or prevents them from doing so. There is some research regarding various motives and barriers for PA during the entirety of pregnancy [14-16]; however, these studies do not examine motives and barriers by each individual trimester. In addition, to the authors' knowledge, there are no data regarding motives and barriers to exercising in women who are affiliated with a health club. This group should be examined further for 2 primary reasons. First, women who belong to a health club may have different motives and barriers to exercising throughout pregnancy as they have already made a conscious decision to be a paying member. Second, women in a health club already value being physically active so helping them stay active throughout pregnancy by reducing barriers is important. In a recent review of perceived barriers to leisure-time PA during pregnancy, qualitative data showed 5 primary themes emerging as barriers: (1) pregnancy-related symptoms and limitation, (2) time constraints, (3) perceptions of already being active, (4) lack of motivation, and (5) mother-child safety concerns [17]. However, these data were assessed over the entirety of pregnancy and not by individual trimesters. Pregnancyrelated symptoms (such as nausea, energy levels, and discomfort due to anatomical changes from the growing foetus) change over the course of gestation, thus a more detailed analysis of motives and barriers by trimester is important. Studying trimester-specific barriers to exercise could help healthcare and fitness professionals tailor specific PA messages. Therefore, the purpose of this study was to describe the most frequent motives for and barriers to maternal exercise by trimester in exercising and non-exercising women who belonged to a health club.

\section{Material and methods}

A retrospective, cross-sectional design was implemented using a convenience sample. The participants included women who were over 18 years old, spoke/ read English, and had given birth in the past 5 years. The women were asked to consider their most recent pregnancy if they had been pregnant more than once during the previous 5 years. They also had to belong to Anytime Fitness $^{\circledR}$ and/or Anytime Health ${ }^{\mathrm{TN}}$. Anytime Fitness ${ }^{\circledR}$ is a 24 -hour health club that offers a variety of cardiovascular equipment and resistance training machines (i.e., Nautilus, Life Fitness, Star Trac, and Precor), with the average cost of membership being $\$ 39 /$ month. In addition to the facilities, members have access to Anytime Health ${ }^{\mathrm{nn}}$, the official wellness website of Anytime Fitness ${ }^{\circledast}$. Anytime Fitness ${ }^{\circledR}$ was chosen as the health club for recruitment because the study was part of a larger project investigating resistance training during pregnancy; thus, we needed to target women who were likely to apply resistance training during their pregnancies. In addition, the Anytime Fitness ${ }^{\circledR}$ directors were willing to advertise and promote the study across the United States, which allowed for a geographically diverse sample. An incentive was not offered to complete the survey.

The participants were recruited via posters displayed in Anytime Fitness ${ }^{\circledR}$ facilities across the United States. The posters directed women to the Anytime Health $^{\text {mi }}$ website, housing the online survey, which took approximately 20-30 min to complete. The survey was developed primarily to assess resistance training, aerobic exercise, and activities of daily living during each trimester of pregnancy and the postpartum period. It also included questions regarding motives for and barriers to exercising near the end. Each woman was asked to provide 3 motives for and 3 barriers to exercise for each trimester of their most recent pregnancy if they were pregnant more than once in the last 5 years.

The women were first asked, 'What was/were your motivation/s to exercise during pregnancy?' Each woman then chose her first, second, and third motive for exercise for each trimester, thus giving a total of 9 motives (i.e., 3 motives per trimester). The women were able to choose from a pre-determined drop-down list of 9 motives, which included: fitness, tone and strength, weight control, relieve stress, enjoyment, have a regular routine, stay healthy, be with friends/socialize, and other. The motives were predetermined and worded exactly as listed above, on the basis of previous literature $[2,14,18]$.

The women were then asked, 'What were the top 3 barriers that you think interfered with your ability to participate in regular physical activity during pregnancy?' Each woman chose her first, second, and third barrier to exercise for each trimester, thus giving a total of 9 barriers (i.e., 3 barriers per trimester). A total of 27 barriers were also in a drop-down menu and included: lack of time/too busy, lack of motivation, lack of child- 
care, lack of energy/tiredness, pregnancy-related illness (e.g., nausea), non-pregnancy-related illness (e.g., cold/ flu), on bed rest/doctor told me, dislike exercise, weather, work/work time, scheduling, wanting to spend time with children, injury, lack of sleep, child preparation time, laziness/excuses, family demands/housework, accessibility, money, boredom, guilt, apathy/lethargy, felt too unwell, exercise was not safe, unsure what exercise was safe, exercise was uncomfortable, and none. The barriers were predetermined and worded exactly as listed in previous literature $[2,14,18,19]$.

A Physical Activity Survey was developed to assess various forms of exercise, emphasizing resistance training, during pregnancy and the postpartum period. Various domains of maternal exercise were queried, such as resistance training, as well as light, moderate, and vigorous aerobic exercise. Most exercise questions were modified from the National Health and Nutrition Examination Survey 2005-2006 [20]. Overall, the primary modifications from the survey were changes in the introductory segment, which was to reflect the specific time point in pregnancy and only ask about leisure-time exercise rather than all activities. If a woman responded that she had performed any exercise in her leisure time during pregnancy, she then was questioned about the frequency, intensity, duration, and type of exercise performed.

Assumptions were not made regarding the participants' activity levels even though they needed to be paying members to Anytime Fitness ${ }^{\circledR}$ in order to be included in the study. Therefore, 2 groups were created depending on exercise status: (1) 'some exercise' included women who reported participating in resistance training, light-intensity aerobic exercise, moderate-intensity aerobic exercise, or vigorous-intensity aerobic exercise at any time point during pregnancy (i.e., a woman might have exercised during the first trimester, but then did not exercise during the remainder of pregnancy); and (2) 'no exercise' included women who reported none of the above (i.e., women who did not partake in any structured exercise throughout the entirety of her pregnancy, however, belonged to Anytime Fitness ${ }^{\circledast}$ and/or was part of the Anytime Health ${ }^{\mathrm{m}}$ website). The exercise groups were classified in this way because all women who engaged in resistance training also reported aerobic exercise; therefore, the impact of resistance training alone could not be assessed. Thus, the study participants were classified to the 'some exercise' or 'no exercise' group for both prepregnancy and pregnancy. Demographic questions, including those referring to the number of months postpartum, were asked at the very end of the survey.
Descriptive statistics were computed. Frequencies were calculated to determine the first, second, and third most commonly reported motives for and barriers to exercise for each trimester. Analyses also included chi-squared tests for proportions of the most commonly reported top motives and barriers by trimester to establish if there was a statistically significant difference by either pre-pregnancy or pregnancy exercise status as pre-pregnancy exercise levels affect pregnancy exercise levels. Fisher exact tests were performed for measures that had cell counts $<5$. The level of statistical significance was set as $\alpha=0.05$. All statistical analyses were completed by using the IBM SPSS Statistics software, version 21.

\section{Ethical approval}

The research related to human use has complied with all the relevant national regulations and institutional policies, has followed the tenets of the Declaration of Helsinki, and has been approved by the Biomedical Institutional Review Board of Michigan State University.

\section{Informed consent}

Informed consent has been obtained from all individuals included in this study.

\section{Results}

Over the course of approximately 2 years, 695 women clicked on the survey link. However, many did not start and/or complete the whole survey (time to complete was approximately 20-30 min), leaving the analytic sample of 295 women. The participants were predominantly white (not Hispanic, not Latino), married, college or post-graduate educated, and reported an annual household income of \$50,000-74,999 (Table 1). The recall timeframe was up to 5 years; however, $75.4 \%$ of women who completed the survey had a child aged 2 years or less (Table 1 ). The time of the survey completion ranged from as little as 1 week $(n=1)$ to 5 years post-delivery $(n=5)$. Among those who exercised in trimester 1, women declared approximately 25-30 min of resistance training 3 days/week, 25$30 \mathrm{~min}$ of light-intensity aerobic activity 5 days/week, 30-35 min of moderate-intensity aerobic activity 5 days/week, and 40-45 min of vigorous aerobic activity 3 days/week. In trimester 2, the women reported approximately 25 min of resistance training 3 days/ week, 25-30 min of light-intensity aerobic activity, 30-35 min of moderate-intensity aerobic exercise 4 days/week, and 35-40 min of vigorous-intensity aero- 


\section{HUMAN MOVEMENT}

E. White, K. Pfeifer, C. Holzman, J. Pivarnik, Exercise motives and barriers in pregnancy

Table 1. Demographic characteristics of the sample by pre-pregnancy exercise status

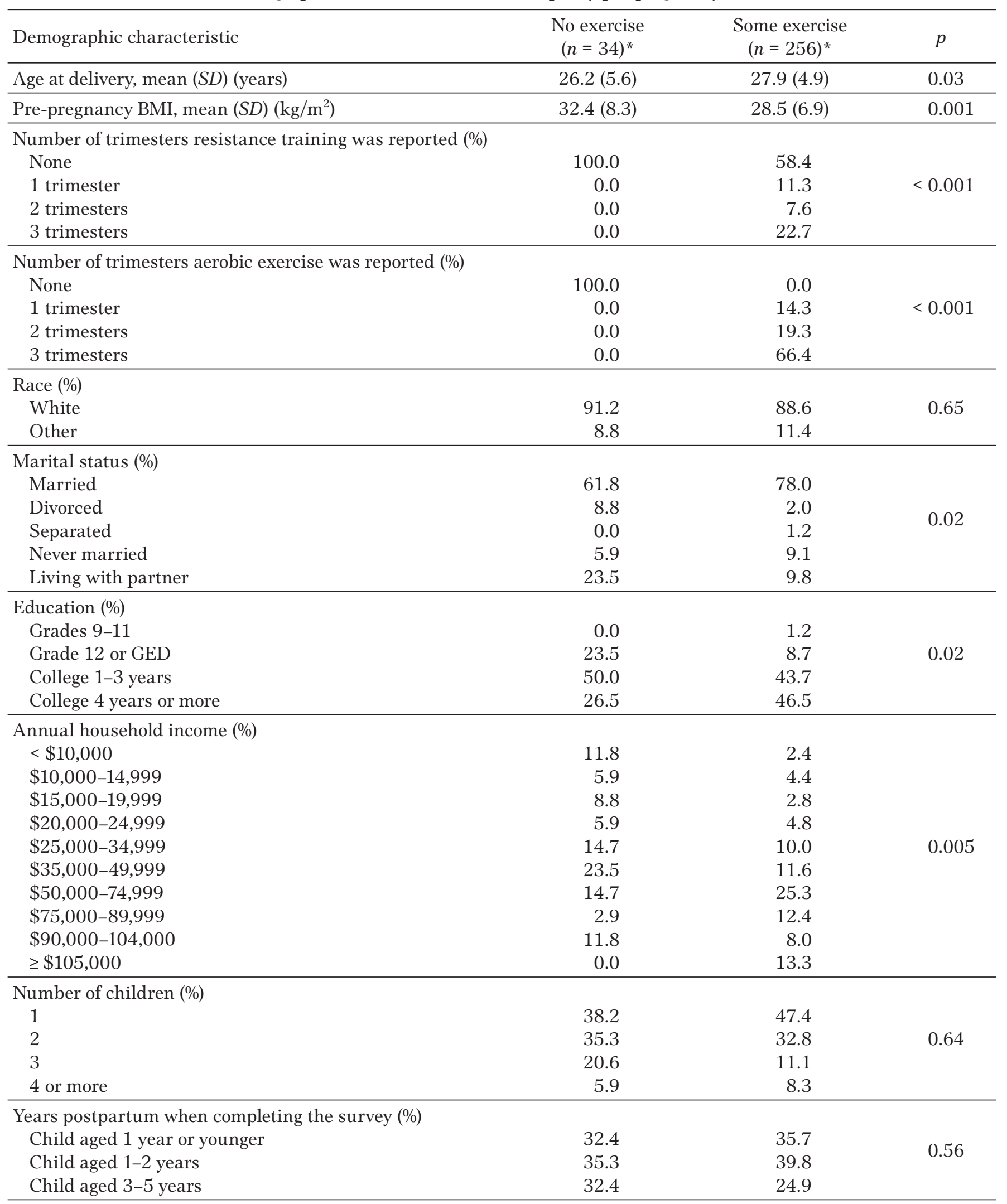

SD - standard deviation, BMI - body mass index, GED - General Education Development

* A total of 295 responses were usable, but because participants did not answer every demographic question, sample sizes depended on the actual number of responses. 
Table 2. Percentage of women $(n=295)$ responding to top 3 motives to exercise during pregnancy by trimester.

Motives are listed from most to least prevalent by the primary motive in each trimester

\begin{tabular}{lccc}
\hline \multicolumn{4}{c}{ Trimester 1} \\
\hline Motives & $1^{\text {st }}$ motive & $2^{\text {nd }}$ motive & $3^{\text {rd }}$ motive \\
\hline Stay healthy & 37.4 & 20.2 & 12.4 \\
Weight control & 19.8 & 27.0 & 21.2 \\
Fitness & 13.7 & 15.9 & 10.1 \\
Other & 6.5 & 3.9 & 3.7 \\
Be with friends/socialize & 6.5 & 2.6 & 5.5 \\
Have a regular routine & 5.3 & 7.3 & 9.7 \\
Enjoyment & 5.3 & 6.0 & 9.2 \\
Relieve stress & 4.2 & 10.7 & 21.7 \\
Tone and strength & 1.1 & 6.4 & 6.5 \\
\hline
\end{tabular}

\begin{tabular}{lccc}
\hline \multicolumn{4}{c}{ Trimester 2} \\
\hline Motives & $1^{\text {st }}$ motive & $2^{\text {nd }}$ motive & $3^{\text {rd }}$ motive \\
\hline Stay healthy & 35.5 & 22.2 & 15.0 \\
Weight control & 27.1 & 26.7 & 18.9 \\
Fitness & 11.6 & 12.2 & 9.7 \\
Other & 6.8 & 4.5 & 4.9 \\
Relieve stress & 6.4 & 13.6 & 21.8 \\
Be with friends/socialize & 4.8 & 1.4 & 6.3 \\
Have a regular routine & 4.4 & 7.7 & 10.2 \\
Enjoyment & 2.4 & 5.9 & 8.7 \\
Tone and strength & 1.2 & 5.9 & 4.4 \\
\hline
\end{tabular}

\begin{tabular}{lccc}
\hline \multicolumn{4}{c}{ Trimester 3} \\
\hline Motives & $1^{\text {st }}$ motive & $2^{\text {nd }}$ motive & $3^{\text {rd }}$ motive \\
\hline Stay healthy & 32.9 & 20.3 & 13.2 \\
Weight control & 25.5 & 30.7 & 19.3 \\
Other & 11.5 & 8.5 & 11.2 \\
Relieve stress & 10.7 & 16.5 & 16.2 \\
Fitness & 8.2 & 7.5 & 9.1 \\
Have a regular routine & 4.9 & 3.8 & 10.2 \\
Be with friends/socialize & 3.7 & 1.9 & 6.1 \\
Enjoyment & 1.6 & 7.1 & 9.6 \\
Tone and strength & 0.8 & 3.8 & 5.1 \\
\hline
\end{tabular}

bic exercise 3 days/week. In trimester 3, the participants applied approximately 25-30 min of resistance training 2.5 days/week, 25-30 min of light-intensity aerobic activity 5 days/week, 30-35 min of moderateintensity aerobic activity 3.5 days/week, and $40-45 \mathrm{~min}$ of vigorous aerobic activity 2.5 days/week.

\section{Motives for exercise during pregnancy}

The most salient motive for exercise during all 3 trimesters of pregnancy was to stay healthy. The most commonly reported second motive was weight control
Table 3. Chi-squared test of proportions and probability values $(p)$ of the proportion of women

who pointed at 'stay healthy' as the top motive and 'lack of energy' as the top barrier in each trimester

by exercise status ('no exercise' or 'some exercise') during pre-pregnancy and pregnancy

\begin{tabular}{|c|c|c|c|}
\hline \multicolumn{4}{|c|}{ Pre-pregnancy exercise status } \\
\hline Top motive/barrier & $\begin{array}{c}\text { No exercise } \\
\%(n)\end{array}$ & $\begin{array}{c}\text { Some exercise } \\
\%(n)\end{array}$ & $p$ \\
\hline \multicolumn{4}{|l|}{ Stay healthy } \\
\hline Trimester 1 & $27.78(5)$ & $38.43(93)$ & 0.37 \\
\hline Trimester 2 & $30.00(6)$ & $36.24(83)$ & 0.58 \\
\hline Trimester 3 & $25.00(5)$ & $33.64(74)$ & 0.43 \\
\hline \multicolumn{4}{|l|}{ Lack of energy } \\
\hline Trimester 1 & $12.50(4)$ & $28.57(70)$ & $0.06 *$ \\
\hline Trimester 2 & $10.00(3)$ & $19.43(48)$ & $0.32 *$ \\
\hline Trimester 3 & $16.67(5)$ & $19.11(47)$ & 0.75 \\
\hline \multicolumn{4}{|c|}{ Exercise status during pregnancy } \\
\hline Top motive/barrier & $\begin{array}{c}\text { No exercise } \\
\%(n)\end{array}$ & $\begin{array}{c}\text { Some exercise } \\
\%(n)\end{array}$ & $p$ \\
\hline \multicolumn{4}{|l|}{ Stay healthy } \\
\hline Trimester 1 & $32.35(11)$ & $37.95(85)$ & 0.53 \\
\hline Trimester 2 & $33.33(11)$ & $35.51(76)$ & 0.81 \\
\hline Trimester 3 & $33.33(11)$ & $32.52(67)$ & 0.93 \\
\hline \multicolumn{4}{|l|}{ Lack of energy } \\
\hline Trimester 1 & $21.28(10)$ & $28.82(66)$ & 0.29 \\
\hline Trimester 2 & $15.91(7)$ & $19.40(45)$ & 0.59 \\
\hline Trimester 3 & $18.18(8)$ & $20.00(46)$ & 0.78 \\
\hline
\end{tabular}

* Fisher's exact test

in all 3 trimesters and the most commonly reported third motive was to relieve stress in the first and second trimesters and to control weight in the third trimester (Table 2). Some women who responded 'other' completed the supplementary question to explain. The reasons that the women provided as 'other' included: did not want to exercise/no motivation to exercise $(n=17)$, recommendation from midwife/physician and/or a complication with her pregnancy $(n=11)$, to induce labour $(n=6)$, to ease back pain $(n=2)$, and to improve flexibility $(n=2)$.

When the participants were classified by pre-pregnancy exercise status, it turned out that $88.3 \%(n=$ 256) participated in resistance training, light-, moderate-, or vigorous-intensity aerobic exercise, while $11.7 \%(n=34)$ did not. The proportion of women reporting 'stay healthy' as the top motive for exercise was not significantly different between exercisers and non-exercisers during pre-pregnancy or pregnancy (Table 3). 


\section{HUMAN MOVEMENT}

E. White, K. Pfeifer, C. Holzman, J. Pivarnik, Exercise motives and barriers in pregnancy

Table 4. Percentage of women $(n=295)$ pointing at top 3 barriers to exercise during pregnancy by trimester. Barriers are listed from most to least prevalent by the primary barrier in each trimester

\begin{tabular}{|c|c|c|c|c|c|c|c|c|}
\hline \multicolumn{3}{|c|}{ Trimester 1} & \multicolumn{3}{|c|}{ Trimester 2} & \multicolumn{3}{|c|}{ Trimester 3} \\
\hline $1^{\text {st }}$ & $2^{\text {nd }}$ & $3^{\text {rd }}$ & $1^{\text {st }}$ & $2^{\text {nd }}$ & $3^{\text {rd }}$ & $1^{\text {st }}$ & $2^{\text {nd }}$ & $3^{\text {rd }}$ \\
\hline barrier & barrier & barrier & barrier & barrier & barrier & barrier & barrier & barrier \\
\hline \multicolumn{3}{|c|}{ Lack of energy/tiredness } & \multicolumn{3}{|c|}{ Lack of energy/tiredness } & \multicolumn{3}{|c|}{ Lack of energy/tiredness } \\
\hline 27.0 & 19.1 & 8.3 & 18.9 & 10.9 & 14.2 & 19.4 & 20.6 & 11.0 \\
\hline \multicolumn{3}{|c|}{ Pregnancy-related illness } & \multicolumn{3}{|c|}{ None } & \multicolumn{3}{|c|}{ Exercise was uncomfortable } \\
\hline 18.5 & 8.9 & 6.9 & 11.4 & 8.7 & 13.7 & 17.2 & 10.3 & 13.0 \\
\hline \multicolumn{3}{|c|}{ None } & \multicolumn{3}{|c|}{ Lack of time/too busy } & \multicolumn{3}{|c|}{ Bed rest/physician told me } \\
\hline 11.7 & 10.2 & 15.1 & 9.6 & 8.3 & 6.4 & 6.8 & 1.3 & 2.5 \\
\hline \multicolumn{3}{|c|}{ Lack of time/too busy } & \multicolumn{3}{|c|}{ Lack of motivation } & \multicolumn{3}{|c|}{ Lack of time/too busy } \\
\hline 9.3 & 7.7 & 8.7 & 8.2 & 11.3 & 6.4 & 6.5 & 4.0 & 7.5 \\
\hline \multicolumn{3}{|c|}{ Lack of motivation } & \multicolumn{3}{|c|}{ Laziness/excuses } & \multicolumn{3}{|c|}{ Lack of motivation } \\
\hline 5.7 & 8.5 & 7.3 & 7.8 & 6.1 & 9.8 & 6.1 & 11.7 & 9.0 \\
\hline \multicolumn{3}{|c|}{ Laziness/excuses } & \multicolumn{3}{|c|}{ Work/work time } & \multicolumn{3}{|c|}{ None } \\
\hline 5.3 & 5.1 & 9.2 & 7.5 & 10.4 & 5.4 & 5.7 & 6.7 & 9.5 \\
\hline \multicolumn{3}{|c|}{ Felt too unwell } & \multicolumn{3}{|c|}{ Pregnancy-related illness } & \multicolumn{3}{|c|}{ Laziness/excuses } \\
\hline 3.9 & 4.3 & 1.8 & 5.3 & 2.2 & 2.5 & 5.3 & 5.4 & 11.5 \\
\hline \multicolumn{3}{|c|}{ Work/work time } & \multicolumn{3}{|c|}{ Lack of childcare } & \multicolumn{3}{|c|}{ Pregnancy-related illness } \\
\hline 2.8 & 6.4 & 6.4 & 3.2 & 3.9 & 0.5 & 4.7 & 3.1 & 0.5 \\
\hline \multicolumn{3}{|c|}{ Lack of childcare } & \multicolumn{3}{|c|}{ Family demands/housework } & \multicolumn{3}{|c|}{ Lack of sleep } \\
\hline 2.5 & 3.4 & 1.8 & 3.2 & 5.2 & 6.4 & 4.3 & 9.9 & 6.0 \\
\hline \multicolumn{3}{|c|}{ Unsure what exercise was safe } & \multicolumn{3}{|c|}{ Felt too unwell } & \multicolumn{3}{|c|}{ Work/work time } \\
\hline 2.1 & 2.1 & 5.5 & 2.5 & 0.4 & 2.9 & 3.6 & 3.6 & 3.5 \\
\hline
\end{tabular}

\section{Barriers to exercise during pregnancy}

The most common barrier to exercise across all 3 trimesters was 'lack of energy/tiredness'. The most commonly reported second barrier was 'lack of energy' during trimester 1, 'lack of motivation' during trimester 2, and 'lack of energy' during trimester 3 . The most frequently enumerated third barrier was 'none' during trimester 1 , 'lack of energy' during trimester 2 , and 'exercise was uncomfortable' during trimester 3 (Table 4). During all 3 trimesters, very few women $(<2 \%)$ reported the following barriers to exercise: dislike exercise, weather, injury, child preparation time, accessibility, money, guilt, or apathy/lethargy.

When the participants were classified by exercise status, the proportion of women reporting 'lack of energy' as the top barrier to exercise was not signifi- cantly different between exercisers and non-exercisers during pre-pregnancy or pregnancy (Table 3).

\section{Discussion}

Overall, the purpose was to describe the most frequent motives for and barriers to maternal exercise by trimester in exercising and non-exercising women who belonged to a health club. Owing to the known benefits of PA during pregnancy [1], exploring motives and barriers by trimester to keep active women active during pregnancy was important. In this study, PA motives and barriers did not change statistically significantly by trimester regardless of exercise status $(p>0.05)$. However, changes in specific motives and barriers did emerge (such as 'exercise to relieve stress' and 'exercise became too uncomfortable') as pregnancy 
continued. Thus, tailoring PA messages to overcome the most salient barriers in each trimester, such as education regarding how to modify exercises in the third trimester to make exercise more comfortable, may be more effective than promoting general health benefits of PA during pregnancy.

\section{Motives for exercise during pregnancy}

The most commonly reported motives for exercise were to stay healthy, control weight, and relieve stress. This is consistent with previous research $[2,10,14]$. While it was expected that the motives may change throughout pregnancy, overall, there were no statistically significant changes among trimesters. Thus, our results suggest that the investigated women were well aware of the benefits of exercise since the top motive for exercise was 'stay healthy,' which was further reflected in the high percentage of women participating in some exercise throughout pregnancy. However, these results should be interpreted with caution since the current sample consisted of women who already belonged to the Anytime Fitness ${ }^{\circledast}$ facility and/or adjoining website (owing to the primary purpose of the original study); therefore, health and fitness were already a high priority in this particular group of women. It is assumed that the investigated group perceived exercise as a valuable and necessary component of health even before pregnancy. This finding is similar to that observed in a group of 17 women who performed regular resistance training before becoming pregnant and continued during pregnancy [21]. The major motive for continuing resistance training during pregnancy was the expected benefits - to both mother and foetus. All of the women stated that there were more health benefits than risks and some used exercise as motivation to avoid common pregnancy-related issues such as postural changes and back pain [21]. Thus, while these women did not describe motives by each trimester, staying healthy was a top priority and corroborated the current findings.

The awareness that exercise is beneficial during pregnancy may be unique to this group of women considering that many were college-educated. Melton et al. [22] reported that in a sample of 88 American rural pregnant women (60.2\% white), only $56.8 \%$ were convinced that they could exercise without consulting a physician and $61.4 \%$ knew that exercise could help prevent or treat gestational diabetes. Related, in a study of 57 nulliparous pregnant women, $81 \%$ received some type of advice on PA at 16 weeks gestation; however, $67 \%$ of that group obtained most of their infor- mation from books and magazines, while $47 \%$ from friends and family, compared with only $18 \%$ from health professionals [23]. In addition, 2 different studies showed that factors toward beliefs about exercise varied most by education level, race/ethnicity, and regular exercise participation [15, 24]. Thus, informing women who do not belong to a health club about the benefits of exercise during pregnancy, especially early in pregnancy, may play a key role in motivating them to exercise during pregnancy. Subsequently, if women do not know the benefits of exercise and/or know if they can exercise without physician clearance, the motive to exercise to 'stay healthy' most likely will not play a driving role in motivating them to be active. Therefore, the current results, showing that to 'stay healthy' is the top motivation to exercise during pregnancy, may be unique to the analysed sample, who already belonged to a health club and/or the adjoining website.

In the current investigation, only $1.1 \%$ of women thought that exercise was unsafe during the first and second trimester, $2.5 \%$ believed that exercise was unsafe during the third trimester, and approximately $2 \%$ were unsure of what exercises were considered safe. Consequently, the sample involved in the present investigation appears to be much more knowledgeable about the benefits of exercise during pregnancy than women who do not belong to a fitness facility. The greater knowledge also could be explained by the high percentage (approximately 75\%) of women within the sample having at least some college education.

It is also of note that motives did not differ statistically significantly by pre-pregnancy or pregnancy exercise status. While regular participation in exercise in the pre-pregnancy period is often associated with higher levels of exercise during pregnancy [15, 24], in the present sample, there were no differences in the motives in each trimester by exercise status. As indicated previously, this could be unique to the current sample, who already belonged to a health club and/or the adjoining website.

\section{Barriers to exercise during pregnancy}

Choosing to regularly participate in exercise or not is dependent on a variety of factors; however, findings from a systematic literature review stated that pregnancy, itself, was negatively associated with overall PA and exercise [25]. Regardless of trimester, pregnancy brings new barriers to exercise that women have not experienced before, even in regular exercisers prior to pregnancy, thus examining barriers by trimester could lead to maintaining levels of PA throughout 
E. White, K. Pfeifer, C. Holzman, J. Pivarnik, Exercise motives and barriers in pregnancy

pregnancy. In the current study, the most commonly reported first barrier during all trimesters was lack of energy. Other highly indicated barriers were pregnancy-related illness during the first trimester and exercise being too uncomfortable during the third trimester. These results are consistent with previous research $[8,10,16,18,26]$.

During the second trimester, lack of energy, lack of time, lack of motivation, work, and laziness all emerged as top barriers, which is in line with previous research. In a previous study of 52 underserved urban pregnant women, the most commonly reported barrier to exercise was employment obligations (25.8\%) [27]. The authors observed that $93.5 \%$ of women did not participate in a Zumba class, even though 52\% showed interest in taking the class; however, women reported that they would be more likely to exercise if provided a DVD exercise video for home use [27]. Thus, as an alternative of health-care providers espousing the benefits of exercise, it may be more beneficial to provide information regarding other aspects of life that tend to be barriers. For example, referring patients to seek information on time-management and/or providing alternative and more time-efficient methods to exercise (i.e., DVDs) may be more beneficial and effective for promoting PA.

During the third trimester, the most commonly reported barrier was exercise becoming uncomfortable, which is also consistent with previous research [18]. Because of the growing foetus and therefore changes in centre of gravity, one possible way to overcome a woman's discomfort is by helping her find support belts and/or suggesting a variety of modalities (i.e., free weights, weight machines, resistance bands, exercise balls, group exercise classes, etc.) to accommodate her changing body. Not only does pregnancy alter a woman's centre of gravity, but also other physical changes such as nausea, back/pelvic pain, and contractions can limit a woman's ability to feel comfortable and be active [21]. Providing education on how to modify exercises and/or switch modalities may help women find ways to continue exercise in the third trimester, as their body is changing. Other suggestions include information regarding exercises to strengthen the surrounding musculature in order to reduce back/ pelvic pain and allow women to maintain activity levels [21]. Offering a variety of exercises may help the mother exercise in a safer and more comfortable manner as she can alter her exercise routine to meet the changes she is undergoing.

In our analyses, barriers did not differ by pre-pregnancy or pregnancy exercise status. We chose to ex- amine this since pre-pregnancy activity levels often influence the amount of activity performed during pregnancy $[15,24]$. Thus, to explain these results, we hypothesize that women already paying for a gym membership may overcome the lack of energy more readily than those who are not paying gym members.

Surprisingly, lack of childcare did not emerge as a primary barrier throughout all of pregnancy in the current sample. Previous studies have reported lack of childcare as a main barrier to exercise [27, 28]. One reason for this was that approximately half of the women in our sample were primiparas; therefore, childcare was not an issue during their pregnancy. Of the multiparous women (54.1\%), it is possible that childcare was not a barrier because they made paying for a gym membership a priority and thus took appropriate measures to ensure they could use it, which included finding childcare.

While our study participants' top barrier did not change significantly by trimester, it is important that healthcare and fitness professionals understand how different barriers emerge in each trimester so as to tailor their activity recommendations. For instance, if a woman stated that she lacked energy to be active, the healthcare provider could promote the immediate benefits of being active rather than the long-term ones. This was found in a study of 226 midlife women (40-60 years old), in which those who had exercise goals related to distant factors such as health and healthy aging were less likely to exercise than those whose exercise goals related to daily life, even though both goals were equally valued [29]. The authors suggested that if exercise was 'rebranded' as a way to immediately feel better rather than focusing on more long-term goals (i.e., healthy overall pregnancy, reduced risk of gestational diabetes, easier labour, etc.), healthcare providers and health professionals may be more effective in promoting their message. Further, according to Melton et al. [22], only $21.6 \%$ of pregnant American rural women knew that exercise could increase energy levels. Thus, it may be possible to inform women about the immediate energizing effects of exercise to help overcome the most commonly reported barrier of lacking energy.

\section{Limitations}

There were several limitations to this study. First, the data were self-reported and collected retrospectively. There may be an inherent error when asking women to recall specific motives and barriers by trimester up to 5 years postpartum. Previous research 
has shown that women can accurately recall trimesterspecific PA up to 6 years postpartum with moderate to high validity [30], thus we assume that our study's 5 -year time frame was acceptable for recalling trimester-specific motives and barriers. In addition, 75.4\% of women completed the survey when their pregnancy of interest was within 2 years, giving potentially less recall bias. The most limiting factor is that all study participants belonged to a fitness facility (or were an online member) and thus the results are not generalizable to women who do not have a gym membership. Further, the participants were primarily white, married, college or post-graduate educated, and had an annual household income of \$50,000-74,999. Thus, these results should be confirmed in a larger and more diverse sample including women without a health club membership.

\section{Conclusions}

In conclusion, the primary motive for exercise in pregnancy among women who belonged to a fitness centre was to stay healthy and the primary barrier was lack of energy in each of the 3 trimesters. This held true regardless of exercise status during pregnancy, and may therefore apply to pregnant women without a gym membership. While the motives and barriers did not change statistically significantly by trimester, other motives and barriers did emerge (such as 'exercise to relieve stress' and 'exercise became too uncomfortable') as pregnancy continued. Therefore, having healthcare and fitness professionals tailor their messages regarding how women can overcome trimesterspecific barriers is important, especially during the third trimester, when PA declines the most. Rather than focusing PA messages on longer-term benefits, women may find more relevance to the immediate effects of exercise, such as feeling more energized, especially since that is the primary barrier to exercise.

\section{Acknowledgements}

The authors would like to acknowledge Mark Daly and Brian Zehetner of Anytime Fitness ${ }^{\circledR}$ for their assistance.

\section{Disclosure statement}

This work was supported by a Student Research Award from Blue Cross Blue Shield of Michigan. No author has any financial interest or received any financial benefit from this research.

\section{Conflict of interest}

The authors state no conflict of interest.

\section{References}

1. U.S. Department of Health and Human Services. 2008 Physical Activity Guidelines for Americans. U.S. Department of Health and Human Services: Washington D.C.; 2008.

2. Symons Downs D, Hausenblas H. Women's exercise beliefs and behaviors during their pregnancy and postpartum.JMidwiferyWomens Health. 2004;49(2):138144; doi: 10.1016/j.jmwh.2003.11.009.

3. American College of Obstetricians and Gynecologists. Exercise During Pregnancy. American College of Obstetricians and Gynecologists: Washington D.C.; 2003.

4. Brown WJ, Mishra G, Lee C, Bauman A. Leisure time physical activity in Australian women: relationship with well being and symptoms. Res Q Exerc Sport. 2000;71(3):206-216; doi: 10.1080/02701367.2000. 10608901.

5. Rice PL, Fort IL. The relationship of maternal exercise on labor, delivery and health of the newborn. J Sports Med Phys Fitness. 1991;31(1):95-99.

6. Shangold MM. Exercise during pregnancy: current state of the art. Can Fam Physician. 1989;35:1675-1680.

7. Work JA. Is weight training safe during pregnancy? Phys Sportsmed. 1989;17(3):256-259; doi: 10.1080/ 00913847.1989.11709749.

8. Gaston A, Cramp A. Exercise during pregnancy: a review of patterns and determinants. J Sci Med Sport. 2011;14(4):299-305; doi: 10.1016/j.jsams.2011.02.006.

9. Hesketh KR, Evenson KR. Prevalence of U.S. pregnant women meeting 2015 ACOG physical activity guidelines. Am J Prev Med. 2016;51(3):e87-e89; doi: 10.1016/ j.amepre.2016.05.023.

10. Haakstad LA, Voldner N, Henriksen T, Bø K. Why do pregnant women stop exercising in the third trimester? Acta Obstet Gynecol Scand. 2009;88(11):12671275; doi: 10.3109/00016340903284901.

11. Oliveira LF, Vieira TM, Macedo AR, Simpson DM, Nadal J. Postural sway changes during pregnancy: a descriptive study using stabilometry. Eur J Obstet Gynecol Reprod Biol. 2009;147(1):25-28; doi: 10.1016/ j.ejogrb.2009.06.027.

12. Moya J, Phillips L, Sanford J, Wooton M, Gregg A, Schuda L. A review of physiological and behavioral changes during pregnancy and lactation: potential exposure factors and data gaps. J Expo Sci Environ Epidemiol. 2014;24(5):449-458; doi: 10.1038/jes. 2013.92.

13. Fell DB, Joseph KS, Armson BA, Dodds L. The impact of pregnancy on physical activity level. Matern Child Health J. 2009;13(5):597-603; doi: 10.1007/s10995008-0404-7.

14. Cioffi J, Schmied V, Dahlen H, Mills A, Thornton C, Duff M, et al. Physical activity in pregnancy: women's 


\section{HUMAN MOVEMENT}

E. White, K. Pfeifer, C. Holzman, J. Pivarnik, Exercise motives and barriers in pregnancy

perceptions, practices, and influencing factors. J Midwifery Womens Health. 2010;55(5):455-461; doi: 10.1016/j.jmwh.2009.12.003.

15. Evenson KR, Bradley CB. Beliefs about exercise and physical activity among pregnant women. Patient Educ Couns. 2010;79(1):124-129; doi: 10.1016/j.pec.2009. 07.028.

16. Weir Z, Bush J, Robson SC, McParlin C, Rankin J, Bell R. Physical activity in pregnancy: a qualitative study of the beliefs of overweight and obese pregnant women. BMC Pregnancy Childbirth. 2010;10:18; doi: 10.1186/1471-2393-10-18.

17. Coll CV, Domingues MR, Gonçalves H, Bertoldi AD. Perceived barriers to leisure-time physical activity during pregnancy: a literature review of quantitative and qualitative evidence. J Sci Med Sport. 2017;20(1): 17-25; doi: 10.1016/j.jsams.2016.06.007.

18. Duncombe D, Wertheim EH, Skouteris H, Paxton SJ, Kelly L. Factors related to exercise over the course of pregnancy including women's beliefs about the safety of exercise during pregnancy. Midwifery. 2009;25(4): 430-438; doi: 10.1016/j.midw.2007.03.002.

19. Cramp AG, Bray SR. Understanding exercise self-efficacy and barriers to leisure-time physical activity among postnatal women. Matern Child Health J. 2011;15(5): 642-651; doi: 10.1007/s10995-010-0617-4.

20. National Health and Nutrition Examination Survey. Physical activity and physical fitness - PAQ; 2006.

21. Petrov Fieril K, Fagevik Olsén M, Glantz A, Larsson M. Experiences of exercise during pregnancy among women who perform regular resistance training: a qualitative study. Phys Ther. 2014;94(8):1135-1143; doi: 10.2522/ptj.20120432.

22. Melton B, Marshall E, Bland H, Schmidt M, Guion W. American rural women's exercise self-efficacy and awareness of exercise benefits and safety during pregnancy. Nurs Health Sci. 2013;15(4):468-473; doi: 10.1111/ nhs.12057.

23. Clarke PE, Gross H. Women's behaviour, beliefs and information sources about physical exercise in pregnancy. Midwifery. 2004;20(2):133-141; doi: 10.1016/j. midw.2003.11.003.

24. Mudd LM, Nechuta S, Pivarnik JM, Paneth N, Michigan Alliance for National Children's Study. Factors associated with women's perceptions of physical activity safety during pregnancy. Prev Med. 2009;49(23):194-199; doi: 10.1016/j.ypmed.2009.06.004.

25. Condello G, Puggina A, Aleksovska K, Buck C, Burns C, Cardon G, et al. Behavior determinants of physical activity across the life course: a "DEterminants of DIet and Physical ACtivity” (DEDIPAC) umbrella systematic literature review. Int J Behav Nutr Phys Act. 2017;14(1): 58; doi: 10.1186/s12966-017-0510-2.

26. Owe KM, Nystad W, Bø K. Correlates of regular exercise during pregnancy: the Norwegian Mother and Child Cohort Study. Scand J Med Sci Sports. 2009;19(5):637645; doi: 10.1111/j.1600-0838.2008.00840.x.
27. Govindappagari S, Lister R, Bernstein PS, Goffman D, Landsberger E. Barriers to exercise for urban parturients. Obstet Gynecol. 2014;123(Suppl. 1):54S; doi: 10.1097/01.AOG.0000447348.67662.79.

28. Pereira MA, Rifas-Shiman SL, Kleinman KP, RichEdwards JW, Peterson KE, Gillman MW. Predictors of change in physical activity during and after pregnancy: project Viva. Am J Prev Med. 2007;32(4):312319; doi: 10.1016/j.amepre.2006.12.017.

29. Segar ML, Eccles JS, Richardson CR. Rebranding exercise: closing the gap between values and behavior. Int J Behav Nutr Phys Act. 2011;8:94; doi: 10.1186/ 1479-5868-8-94.

30. Bauer PW, Pivarnik JM, Feltz DL, Paneth N, Womack CJ. Validation of an historical physical activity recall tool in postpartum women. J Phys Act Health. 2010; 7(5):658-661; doi: 10.1123/jpah.7.5.658. 